\title{
Risikoadaptierte Antikoagulation zur Schlaganfallprävention bei Vorhofflimmern in Deutschland, Österreich und der Schweiz
}

\author{
Analyse des PREFER-in-AF-Registers
}

J. Steffel ${ }^{1 *}$, M. Gwechenberger ${ }^{2 *}$, M. W. H. Müller ${ }^{3}$, B. Ammentorp ${ }^{4}$, H. Darius ${ }^{5}$, P. Kirchhof $f^{6,7}$

\section{Zusammenfassung}

Hintergrund I Das Management von Patienten mit Vorhofflimmern (VHF) hat in den letzten Jahren zahlreiche Verbesserungen erfahren, unter anderem durch die Einführung neuer Scores zur Stratifizierung des Schlaganfallsrisikos, wie auch durch die Verfügbarkeit der nicht-Vitamin-K oralen Antikoagulanzien (NOAK). Ziel der PREFER-inAF-Studie war es, die Versorgung von Patienten mit VHF unter besonderer Berücksichtigung der Schlaganfallprävention durch Antikoagulation zu untersuchen.

Patienten I In Deutschland, Österreich und der Schweiz wurden zwischen Januar 2012 bis Januar 20131771 Patienten in die Studie eingeschlossen (mittleres Alter 71,9 \pm 9,2 Jahre; 63\% Männer). Zu Beobachtungsbeginn betrug die mittlere Zeit seit Erstdiagnose von VHF 4,8 $\pm 5,3$ Jahre, wobei 30,7\% der Patienten paroxysmales, 11,0\% persistierendes, 4,7\% langanhaltend persistierendes, und $53,3 \%$ permanentes VHF aufwiesen. Ein Sinusrhythmus lag bei $25,1 \%$ vor. Der mittlere $\mathrm{CHA}_{2} \mathrm{DS}_{2}$ VASc-Score lag bei 3,7 $\pm 1,8$ Punkten (0 Punkte bei $3,0 \%, 1$ Punkt bei $7,1 \%, \geq 2$ Punkte bei $89,9 \%$ ).

Ergebnisse | Zur Prophylaxe thromboembolischer Ereignisse erhielten 68,1\% der Patienten
Vitamin-K-Antagonisten (VKA, überwiegend Phenprocoumon), 11,6\% wurden mit einem NOAK behandelt (hauptsächlich Rivaroxaban oder Dabigatran), 7,6\% mit Thrombozytenaggregationshemmern (TAH) und 7,7\% mit VKA und TAH in Kombination. Keinerlei Thromboembolie-Prophylaxe erhielten 5,0\% der Patienten. Ein temporäres Absetzen von VKA bei Interventionen wurde bei $29,7 \%$ in den 12 Monaten vor Einschluss berichtet. Die Rate von adäquat eingestellten Patienten (mindestens 2 von 3 INR-Werten im Bereich 2,0-3,0) lag bei 75,1\% (bezogen auf Patienten mit bekannten INR-Werten und Risikoscore).

Eine Blutungsneigung bzw. Blutungen in der Anamnese wurden bei 5,1\% der Patienten berichtet, Hospitalisierungen aufgrund größerer Blutungen in den letzten 12 Monaten bei 1,9\%. Mögliche Risikofaktoren für eine Antikoagulation bestanden bei 76,7\% der Patienten. Der mittlere HAS-BLEDScore betrug 2,1 $\pm 1,1$ Punkte.

Schlussfolgerung I Die Rate der mit oraler Antikoagulation behandelten VHF-Patienten lag mit knapp 90\% deutlich höher als in älteren Beobachtungsstudien. NOAK wurden bei $12 \%$ der Patienten eingesetzt.

\section{Hintergrund}

Vorhofflimmern (VHF) ist die häufigste Rhythmusstörung in der klinischen Praxis. Die Prävalenz des VHF steigt aufgrund der zunehmenden Alterung der Bevölkerung, Zunahme der Risikofaktoren (insbesondere arterielle Hypertonie) und stetigen Fortschritten in der Behandlung kardialer Erkrankungen kontinuierlich an [20]. Patienten mit VHF haben ein stark erhöhtes Risiko für das Auftreten eines ischämischen oder hämorrhagischen Schlaganfalls, sowie für einen frühzeitigen Tod $[1,10]$ Die meisten Patienten mit VHF müssen aufgrund von Risikofaktoren dauerhaft mit Antikoagulanzien behandelt werden, sofern dem keine absoluten Kontraindikationen gegenüberstehen.

In den letzten Jahren hat sich das klinische Management von Patienten mit VHF stetig verbes- sert, besonders hinsichtlich der Antikoagulation. Die nicht-Vitamin-K-abhängigen oralen Antikoagulanzien (NOAK) Dabigatran, Rivaroxaban, Apixaban und Edoxaban zeigten in den großen Zulassungsstudien gegenüber den Vitamin-KAntagonisten (VKA) eine mindestens gleich gute - teilweise sogar überlegene Wirksamkeit bei der Prophylaxe von Schlaganfällen. Darüberhinaus demonstrierten die NOAK ein reduziertes Risiko für Blutungen, vor allem für Hirnblutungen $[5,9,23,24]$. Die VHF-Leitlinien der European Society for Cardiology (ESC) führten 2010 bzw. 2012 einige Neuerungen ein, wie z.B. den $\mathrm{CHA}_{2} \mathrm{DS}_{2}$-VASc zur Abschätzung des Schlaganfallrisikos [4]. Des Weiteren gaben sie bei Patienten mit einem erhöhten Schlaganfallrisiko $\left(\mathrm{CHA}_{2} \mathrm{DS}_{2}\right.$-VASc-Score $\left.\geq 2\right)$ eine klare Empfehlung für eine Antikoagulation beispielsweise mit einem VKA, rieten jedoch bei Patienten ohne thromboembolisches Risiko $\left(\mathrm{CHA}_{2} \mathrm{DS}_{2}\right.$-VASc-
Institute

1 Klinik für Kardiologie, Universitätsspital Zürich, Schweiz

2 Department für Kardiologie, Medizinische Universität, Wien, Österreich

3 Medizinische Abteilung, Daiichi Sankyo Deutschland

4 Daiichi Sankyo Europe, München

5 Innere Medizin - Kardiologie, Angiologie und konservative Intensivmedizin, Vivantes Krankenhaus Neukölln, Berlin

6 University of Birmingham Institute of Cardiovascular Sciences and SWBH NHS Trust, Birmingham, UK

7 Department für Kardiologie und Angiologie, Universitätsklinikum Münster, Deutschland * geteilte Erstautorenschaft

\section{Korrespondenz}

PD Dr. med. Jan Steffel Universitätsspital Zürich Klinik für Kardiologie Rämistrasse 100 CH-8091 Zürich $+41(0) 442551111$ jan.steffel@usz.ch 
Score $=0$ ) zum Verzicht auf eine solche Therapie $[3,4,26]$.

Die PREFER-in-AF-Studie wurde durchgeführt, um das Management von Patienten in diversen europäischen Ländern zu untersuchen [15]. Die Studie wurde kurz nach der Publikation der aktuellen VHF-Leitlinien der ESC initiiert und dokumentiert gleichzeitig die Einführungsphase der NOAK. Um die aktuelle Versorgungslage von Patienten mit VHF in Deutschland, Österreich und der Schweiz (D-A-CH) zu beschreiben, wird im vorliegenden Beitrag über eine vorab spezifizierte Subgruppen-Analyse berichtet.

\section{Methoden}

PREFER-in-AF I „PREvention oF thromboembolic events - European Registry in Atrial Fibrillation“ ist eine internationale, prospektive Registerstudie, die von Januar 2012 bis Januar 2013 den Krankheitsverlauf und die Behandlung von Patienten mit VHF unter Bedingungen der täglichen Praxis dokumentierte [15].

Ethik | Der Beobachtungsplan sowie die Patienten-Einverständniserklärung wurden von der Ethikkommission in Berlin als federführende Institution und von weiteren Ethikkommissionen begutachtet und befürwortet. Alle Patienten erklärten ihr schriftliches Einverständnis vor Studieneinschluss. Das Register wurde entsprechend den Vorgaben der Deklaration von Helsinki durchgeführt. PREFER-in-AF ist im Deutschen Register Klinischer Studien unter der Nummer DRKS00003343 registriert. In der Schweiz wurde die Studie durch die kantonale Ethikkommission bewilligt, in Österreich durch die Ethikkommission der medizinischen Universität Wien positiv votiert.

Auswahl von Ärzten und Patienten I Krankenhausärzte und niedergelassene Ärzte konnten an der Studie teilnehmen, wenn sie in der Behandlung von Patienten mit VHF erfahren waren. Patienten waren für die Teilnahme geeignet, wenn sie eine bestätigte VHF-Diagnose entsprechend der ACC / AHA/ESC-Leitlinien aus dem Jahr 2010 hatten [4], die mittels EKG, Herzschrittmacher oder implantiertem Defibrillator innerhalb der letzten 12 Monate bestätigt worden war. Unbestätigte Verdachtsfälle konnten nicht eingeschlossen werden. Um Verzerrungen durch Selektion der Patienten zu vermeiden, wurden keine expliziten Ausschlusskriterien vorgegeben. Die Patienten sollten in den Zentren konsekutiv eingeschlossen werden.

Datenerfassung und Parameter I Die Patientendaten wurden an zwei Terminen, zu Studienbeginn und nach einem Jahr, dokumentiert. Neben den Basis-Charakteristika der Patienten wurden auch Begleiterkrankungen und Medikamente zur Behandlung des VHF dokumentiert. Der Schwerpunkt der Datenerfassung lag auf der Antikoagulation zur Prävention von Schlaganfall und anderen vaskulären Ereignissen und der Dokumentation von Ereignissen während der Beobachtungszeit.

Datenerfassung und statistische Analyse I Die Daten wurden in ein elektronisches Erfassungsformular eingegeben und über eine sichere Internetverbindung in die Datenbank übertragen. Als qualitätssichernde Maßnahmen wurden

- automatische Plausibilitätsprüfungen bei der Dateneingabe,

- Rückfragen (Queries) basierend auf einem Validierungsplan und

- Monitoring mit Vergleich der Registerdaten mit den Patientenakten

in 5\% der Fälle vorgenommen. Das Studienmanagement wurde durch SSS International Clinical Research GmbH, Germering durchgeführt. Das Register wird durch ein wissenschaftliches Leitungsgremium begleitet.

Die vorliegende Publikation berichtet die Ergebnisse der Eingangsuntersuchung zu Studienbeginn. Für die hier berichteten Analysen wurden die Daten der Patienten aus Deutschland, Österreich und der Schweiz verwendet. Die Daten der Eingangsuntersuchung wurden deskriptiv für die Gesamtkohorte bzw. für Subgruppen ausgewertet. Die Subgruppen wurden eingeteilt nach

- Typ des Vorhofflimmerns,

- Schlaganfallrisiko ( $\mathrm{CHA}_{2} \mathrm{DS}_{2}$-VASc-Score) und

- Blutungsrisiko (HAS-BLED-Score).

Kontinuierliche Variablen werden als Mittelwert mit Standardabweichung bzw. Median (Spannweite) dargestellt, kategorische Variablen als prozentualer Anteil der Patienten mit verfügbaren Werten.

Bei den Analysen zum Erreichen des INR-Zielwertes wurden nur Patienten mit vollständigen Angaben zur INR in den letzten 12 Monaten vor Einschluss (mindestens 3 Werte) ausgewertet. Die statistische Analyse wurde mit SAS Version 9.2. durchgeführt.

\section{Ergebnisse}

Zum Zeitpunkt der Zwischenauswertung vom 11.2.2013 wurden 1771 Patienten aus Deutschland, Österreich und der Schweiz in das Register eingeschlossen. Demografische Angaben und Vor-/Begleiterkrankungen bei Einschluss sind in - Tab. 1 dargestellt. Das mittlere Alter der Patienten lag bei 71,9 $\pm 9,2$ Jahren. Die häufigsten Komorbiditäten der Patienten waren:

- arterielle Hypertonie (81,9\%)

- Diabetes mellitus (31,2\%) 
Tab. 1 Demografische Angaben und Vor-/Begleiterkrankungen bei Einschluss

* Stenose oder Insuffizienz

NSAID: nicht-steroidale Antirheumatika

TAH: Thrombozytenaggregationshemmer.

Für 118 Patienten konnte kein $\mathrm{CHA}_{2} \mathrm{DS}_{2}$-VASc-Score berechnet werden.

\begin{tabular}{|c|c|c|c|c|c|c|c|c|}
\hline & \multicolumn{4}{|c|}{ Typ des VHF } & \multicolumn{3}{|c|}{$\mathrm{CHA}_{2} \mathrm{DS}_{2}$-VASc-Score } & \multirow{2}{*}{$\begin{array}{l}\text { Gesamt } \\
(\mathrm{N}=1771)\end{array}$} \\
\hline & $\begin{array}{l}\text { paroxys- } \\
\text { mal } \\
(n=543)\end{array}$ & $\begin{array}{l}\text { persistie- } \\
\text { rend } \\
(n=195)\end{array}$ & $\begin{array}{l}\text { langanhal- } \\
\text { tend } \\
\text { persistie- } \\
\text { rend }(n=83)\end{array}$ & $\begin{array}{l}\text { perma- } \\
\text { nent } \\
(n=944)\end{array}$ & $\begin{array}{l}0 \\
(n=49)\end{array}$ & $\begin{array}{l}1 \\
(n=117)\end{array}$ & $\begin{array}{l}\geq 2 \\
(n=1487)\end{array}$ & \\
\hline Alter, Jahre & $68,9 \pm 9,8$ & $71,4 \pm 9,6$ & $72,3 \pm 7,8$ & $73,6 \pm 8,3$ & $54,3 \pm 7,6$ & $60,4 \pm 7,5$ & $73,4 \pm 7,9$ & $71,9 \pm 9,2$ \\
\hline Bereich, Jahre & $32-93$ & $26-89$ & $52-87$ & $37-95$ & $26-64$ & $42-74$ & $32-95$ & 26-95 \\
\hline > 65 Jahre, \% & 68,9 & 78,5 & 80,7 & 84,6 & 0 & 23,1 & 86,2 & 78,9 \\
\hline Männer, \% & 60,2 & 63,6 & 65,9 & 64,3 & 100 & 86,3 & 60,0 & 63,0 \\
\hline $\begin{array}{l}\text { Body-Mass-Index } \\
\text { (Mittelwert } \pm S D \text {, } \\
\mathrm{kg} / \mathrm{m}^{2} \text { ) }\end{array}$ & $28,2 \pm 4,6$ & $28,2 \pm 4,9$ & $29,2 \pm 4,9$ & $28,5 \pm 4,9$ & $26,8 \pm 3,2$ & $29,1 \pm 4,9$ & $28,4 \pm 4,8$ & $28,4 \pm 4,8$ \\
\hline $\begin{array}{l}\text { Arterielle Hypertonie, } \\
\%\end{array}$ & 74,8 & 80,6 & 92,3 & 84,9 & 0 & 51,3 & 86,8 & 81,9 \\
\hline Diabetes mellitus, \% & 22,8 & 23,1 & 33,7 & 37,5 & 0 & 1,7 & 35,0 & 31,2 \\
\hline $\begin{array}{l}\text { Adipositas } \\
\left(\text { BMI }>30 \mathrm{~kg} / \mathrm{m}^{2}\right), \%\end{array}$ & 30,0 & 31,8 & 35,9 & 31,4 & 10,2 & 39,3 & 31,2 & 30,8 \\
\hline $\begin{array}{l}\text { Herzklappenfehler, } \\
\% *\end{array}$ & 29,9 & 44,3 & 39,0 & 48,0 & 14,3 & 29,9 & 43,9 & 41,2 \\
\hline $\begin{array}{l}\text { Koronare Herzerkran- } \\
\text { kung, \% }\end{array}$ & 23,9 & 29,2 & 35,9 & 32,2 & 0 & 6,8 & 33,3 & 29,6 \\
\hline Myokardinfarkt, \% & 9,0 & 12,8 & 12,0 & 10,7 & 0 & 0 & 12,2 & 10,5 \\
\hline Schlaganfall, \% & 8,6 & 8,3 & 9,0 & 13,2 & 0 & 0 & 12,2 & 10,7 \\
\hline TIA, \% & 4,0 & 3,1 & 5,1 & 9,4 & 0 & 0 & 8,5 & 7,4 \\
\hline Herzinsuffizienz, \% & 15,8 & 30,2 & 34,6 & 35,4 & 0 & 4,3 & 31,4 & 28,4 \\
\hline $\begin{array}{l}\text { Chronische Nierenin- } \\
\text { suffizienz \% }\end{array}$ & 17,3 & 25,0 & 14,5 & 14,5 & 4,1 & 4,3 & 16,4 & 14,9 \\
\hline $\begin{array}{l}\text { Chronisch obstruktive } \\
\text { Lungenerkrankung \% }\end{array}$ & 7,1 & 9,4 & 7,8 & 11,8 & 4,1 & 2,6 & 10,8 & 9,8 \\
\hline $\begin{array}{l}\mathrm{CHA}_{2} \mathrm{DS}_{2} \text {-VASc-Score, } \\
\text { Mittelwert }\end{array}$ & $3,2 \pm 1,6$ & $3,5 \pm 1,8$ & $3,8 \pm 1,6$ & $4,1 \pm 1,8$ & & $1,0 \pm 0,0$ & $4,1 \pm 1,5$ & $3,7 \pm 1,8$ \\
\hline $\begin{array}{l}\text { HAS-BLED-Score, } \\
\text { Mittelwert }\end{array}$ & $1,9 \pm 1,1$ & $2,1 \pm 1,1$ & $2,4 \pm 1,0$ & $2,2 \pm 1,0$ & $0,4 \pm 0,6$ & $0,9 \pm 0,8$ & $2,2 \pm 1,0$ & $2,1 \pm 1,1$ \\
\hline \multicolumn{9}{|l|}{ EHRA-Score, \% } \\
\hline I & 7,9 & 10,3 & 13,3 & 8,2 & 8,2 & 15,4 & 8,1 & 8,6 \\
\hline II & 45,7 & 35,9 & 33,7 & 47,5 & 44,9 & 43,6 & 44,9 & 45,0 \\
\hline III & 31,7 & 30,8 & 38,6 & 31,7 & 24,5 & 30,8 & 31,9 & 31,8 \\
\hline IV & 10,1 & 15,4 & 13,3 & 9,4 & 14,3 & 6,8 & 11,0 & 10,4 \\
\hline $\begin{array}{l}\text { Blutungen in Vorge- } \\
\text { schichte oder } \\
\text { Prädisposition, \% }\end{array}$ & 4,4 & 3,6 & 6,4 & 5,9 & 0 & 1,7 & 5,8 & 5,1 \\
\hline NSAID oder TAH, \% & 25,2 & 23,1 & 27,7 & 18 & 14,3 & 17,1 & 23,2 & 22,2 \\
\hline Alkoholabusus, \% & 1,3 & 1,6 & 2,6 & 2,7 & 2,0 & 3,4 & 2,1 & 2,0 \\
\hline
\end{tabular}




\begin{tabular}{l} 
Typ des VHF \\
$\begin{array}{l}\text { paroxys- } \\
\text { mal } \\
(n=543)\end{array} \quad(n=195)$ \\
\hline
\end{tabular}

\begin{tabular}{llllll} 
& \multicolumn{3}{c}{$\mathrm{CHA}_{2} \mathrm{DS}_{2}$-VASc-Score } & Gesamt \\
$\begin{array}{llllll}\text { langanhaltend } \\
\text { persistierend } \\
(\mathrm{n}=83)\end{array}$ & $\begin{array}{l}\text { permanent } \\
(\mathrm{n}=944)\end{array}$ & 0 & 1 & $\geq 2$ & $(\mathrm{~N}=1771)$ \\
& & $(\mathrm{n}=49)$ & $(\mathrm{n}=117)$ & $(1487)$ & \\
\hline
\end{tabular}

Einstellung der Patienten bei Einschluss

\begin{tabular}{|c|c|c|c|c|c|c|c|c|}
\hline Sinusrhythmus, \% & 68,9 & 22,8 & 6,1 & 1,9 & 63,3 & 42,7 & 22,6 & 25,1 \\
\hline \multicolumn{9}{|l|}{ Frequenzregulierung, \% } \\
\hline gut (HF 60-100) & 72,6 & 73,8 & 80,7 & 84,0 & 65,3 & 61,5 & 81,0 & 79,1 \\
\hline $\begin{array}{l}\text { akzeptabel (HF } \\
50-59 \text { oder } 101-110)\end{array}$ & 15,8 & 12,3 & 9,6 & 9,7 & 16,3 & 18,8 & 11,0 & 11,9 \\
\hline $\begin{array}{l}\text { schlecht }(H F<50 \\
\text { oder }>110)\end{array}$ & 11,6 & 13,8 & 9,6 & 6,3 & 18,4 & 19,7 & 7,9 & 8,9 \\
\hline \multicolumn{9}{|c|}{ VHF-assoziierte Interventionen in letzten 12 Monaten vor Einschluss } \\
\hline $\begin{array}{l}\text { Medikamentöse } \\
\text { Kardioversion, \% }\end{array}$ & 23,4 & 17,4 & 12,0 & 5,9 & 24,5 & 16,4 & 12,3 & 12,8 \\
\hline $\begin{array}{l}\text { Elektrische Kardio- } \\
\text { version, \% }\end{array}$ & 23,1 & 39,0 & 24,1 & 12,2 & 30,6 & 34,5 & 17,5 & 19,1 \\
\hline Ablation \% & 11,3 & 7,7 & 9,6 & 1,8 & 26,5 & 14,7 & 4,2 & 5,8 \\
\hline $\begin{array}{l}\text { Herzschrittmacher } \\
\text { oder Defibrillator, \% }\end{array}$ & 9,9 & 5,1 & 6,0 & 10,5 & 6,1 & 2,6 & 9,9 & 9,6 \\
\hline
\end{tabular}

Tab. 2 Einstellung der Herzfrequenz bei Einschluss und VHF-assoziierte Interventionen in den letzten 12 Monaten vor Einschluss in die Studie HF: Herzfrequenz.

- Adipositas (30,8\%)

- chronische Niereninsuffizienz (14,9\%)

Z Z.n. Schlaganfall (10,7\%)

- Z.n. Myokardinfarkt (10,5\%)

- chronische obstruktive Lungenerkrankung $(9,8 \%)$

Zu Beobachtungsbeginn betrug die mittlere Zeit seit der VHF-Diagnose 4,8 $\pm 5,3$ Jahre.

- 543 (30,7\%) der Patienten hatten paroxysmales,

- 195 (11,0\%) persistierendes,

- $83(4,7 \%)$ langanhaltend persistierendes und

- 944 (53,3\%) permanentes VHF (für 6 Patienten keine Angaben).

Die Mehrzahl der Patienten wies eine beträchtliche Belastung durch VHF-assoziierte Symptome auf(EHRA-Score III: 31,8\%; EHRA-Score IV: 10,4\%).

Der mittlere $\mathrm{CHA}_{2} \mathrm{DS}_{2}$-VASc-Score lag bei 3,7 $\pm 1,8$ Punkten (0 Punkte bei 3,0\% der Patienten, 1 Punkt bei $7,1 \%, 2+$ Punkte bei $89,9 \%$ ). Die überwiegende Mehrheit der Patienten wies hohe Score-Werte als einen Indikator für ein sehr hohes Schlaganfallrisiko auf. Knapp ein Drittel der Patienten ( $\mathrm{n}=543$; $32,7 \%$ ) hatte einen Score von 5 oder mehr.

25,1\% aller Patienten hatten bei Einschluss einen Sinusrhythmus. Die Einstellung der Herzfrequenz bei Einschluss und VHF-assoziierte Interventionen in letzten 12 Monaten sind in $\mathbf{T a b} .2$ beschrieben. In der Gesamtgruppe hatten $12,8 \%$ der
Patienten eine medikamentöse Kardioversion, 19,1\% eine elektrische Kardioversion, 5,8\% eine Ablation, sowie 9,6\% eine Versorgung mit Herzschrittmacher oder Defibrillator erhalten.

Zur Prophylaxe thromboembolischer Ereignisse erhielten in den 12 Monaten vor Einschluss bzw. zum Zeitpunkt des Einschlusses ( Tab. 3) die Patienten folgende Medikamente:

- 1206 (68,1\%) Patienten VKA als Monotherapie (überwiegend Phenprocoumon),

- 136 ( 7,7\%) VKA in Kombination mit TAH

- $205(11,6 \%)$ NOAK

-5,8\% mit Rivaroxaban

- 5,5\% mit Dabigatran

- 135 (7,6\%) TAH als Monotherapie

Keines der genannten Medikamente zur Thromboembolie-Prophylaxe erhielten 89 (5,0\%) Patienten.

- Tab. 3 zeigt die Verteilung der Antikoagulationsund der antithrombotischen Therapie nach VHFTyp, Abb. 1 nach $\mathrm{CHA}_{2} \mathrm{DS}_{2}$-VASc-Score. Mit zunehmender Punktzahl nahm der Anteil Patienten ohne Antikoagulanzien ab. Auf der anderen Seite erhielten 61,3\% der Patienten mit einem $\mathrm{CHA}_{2} \mathrm{DS}_{2}$ VASc-Score von 0 entweder einen VKA oder NOAK.

Ein temporäres Absetzen von VKA vor therapeutischen oder diagnostischen Interventionen wurde häufig berichtet (29,7\% in den vergangenen 12 Monaten) 


\begin{tabular}{|c|c|c|c|c|c|}
\hline & \multicolumn{5}{|l|}{ Typ des VHF } \\
\hline & $\begin{array}{l}\text { paroxysmal } \\
(n=543)\end{array}$ & $\begin{array}{l}\text { persistierend } \\
(n=195)\end{array}$ & $\begin{array}{l}\text { langanhaltend } \\
\text { persistierend } \\
(\mathrm{n}=83)\end{array}$ & $\begin{array}{l}\text { permanent } \\
(n=944)\end{array}$ & $\begin{array}{l}\text { Gesamt } \\
(N=1771)\end{array}$ \\
\hline TAH Monotherapie & $67(12,3 \%)$ & $18(9,2 \%)$ & $3(3,6 \%)$ & $45(4,8 \%)$ & $7,6 \%$ \\
\hline VKA Monotherapie & $279(51,4 \%)$ & $123(63,1 \%)$ & $50(60,2 \%)$ & $752(79,7 \%)$ & $68,1 \%$ \\
\hline VKA+TAH & $55(10,1 \%)$ & $17(8,7 \%)$ & $9(10,8 \%)$ & $55(5,8 \%)$ & $7,7 \%$ \\
\hline NOAK Monotherapie & $92(16,9 \%)$ & $29(14,9 \%)$ & $14(16,9 \%)$ & $70(7,4 \%)$ & $11,6 \%$ \\
\hline Rivaroxaban & 50 & 17 & 4 & 31 & 102 \\
\hline Dabigatran & 39 & 11 & 10 & 32 & 92 \\
\hline Apixaban & 2 & 0 & 0 & 1 & 3 \\
\hline $\begin{array}{l}\text { Keine der genannten } \\
\text { Gruppen }\end{array}$ & $50(9,2 \%)$ & $8(4,1 \%)$ & $7(8,4 \%)$ & $22(2,3 \%)$ & $5,0 \%$ \\
\hline
\end{tabular}

Anders herum verhielt es sich mit der Antikoagulation hinsichtlich des HAS-BLED-Scores: Je höher der HAS-BLED-Score, desto weniger Patienten erhielten orale Antikoagulanzien ( $\mathbf{A b b}$. 2).

Von den 1342 Patienten unter VKA-Therapie war für $1146(85,4 \%)$ der $\mathrm{CHA}_{2} \mathrm{DS}_{2}$-VASc-Score bekannt und es lagen INR-Werte innerhalb der 12 Monate vor Einschluss vor. Daten dieser Patienten wurden in einer Subgruppen-Analyse ausgewertet. Von den 1146 Patienten hatten

- $16,1 \%$ einen Wert,

- 28,2\% zwei Werte und

- $46,9 \%$ alle drei der letzten drei verfügbaren Werte

innerhalb des Zielbereichs (INR 2,0-3,0). Die Qualität der INR-Einstellung unterschied sich nicht wesentlich, wenn Patienten nach den einzelnen $\mathrm{CHA}_{2} \mathrm{DS}_{2}$-VASc-Kategorien differenziert wurden $(\checkmark$ Abb. 3 ).

Eine Blutungsneigung bzw. Blutungen in der Anamnese wurden bei 5,1\% der Patienten berichtet, wobei Hospitalisierungen aufgrund größerer Blutungen in den letzten 12 Monaten bei 1,9\% angegeben wurden. Mögliche Risikofaktoren für eine Blutung bestanden bei 76,7\% der Patienten (zumeist unzureichend eingestellte Hypertonie, chronische Niereninsuffizienz oder nicht näher bezeichnete Faktoren). Der mittlere HAS-BLEDScore betrug 2,1 $\pm 1,1$ Punkte. Mit zunehmendem Blutungsrisiko nahm der Anteil der mit VKA behandelten Patienten ab, dafür nahm die Gabe von TAH zu.

\section{Diskussion}

In der vorliegenden Analyse der PREFER-in-AFStudie in Deutschland, Österreich und der Schweiz wurde eine orale Antikoagulation bei einer großen Anzahl Patienten eingesetzt. VKA waren zum
Zeitpunkt der Studie mit 68,1\% die am häufigsten verschriebenen Antikoagulanzien. Bei den meisten Patienten wird die Frequenz ausreichend reguliert und rhythmuserhaltende Maßnahmen werden häufig eingesetzt. Dennoch bleiben viele Patienten symptomatisch, was den Bedarf am Einsatz weiterer Therapieoptionen aufzeigt.

Um die Charakteristika der Patienten in unserem Register in einen Kontext vergleichbarer Register zu stellen, eignet sich vor allem das Register des AFNET (5333 Patienten aus Expertenzentren des Kompetenznetzes Vorhofflimmern mit Patienteneinschluss zwischen 2004 bis 2007 [21]) bzw. die ATRIUM-Studie (3667 ambulante Patienten im 2009 [19]). Beide Studien berichteten die Ergebnisse unterteilt nach VHF-Gruppen wie die vorliegende Studie. Im Vergleich zu der hier untersuchten D-A-CH-Kohorte der PREFER-in-AF-Studie waren die Patienten im AFNET im Mittel um 4-5 Jahre jünger (64-71 Jahre), hatten seltener einen Diabetes mellitus (18\%), Herzinsuffizienz (34\%), arterielle Hypertonie (62-66\%), jedoch häufiger eine KHK (29-36\%). In der ATRIUM-Studie betrug das mittlere Alter der Patienten 72 Jahre. Sie hatten einen Diabetes mellitus in 35\%, Herzinsuffizienz in $43 \%$, und KHK in $34 \%$ der Fälle [19]. Der $\mathrm{CHA}_{2} \mathrm{DS}_{2}$-VASc-Score, der die Informationen $\mathrm{zu}$ Alter und Komorbiditäten in einer Kennziffer aggregiert, lag in ATRIUM bei 4,0 $\pm 1,7$ und war somit höher als in unserer Studie. Im Vergleich zum PREFER-in-AF-Gesamtkollektiv (mittleres Alter 71,5 Jahre, 60,1\% Männer, $\mathrm{CHA}_{2} \mathrm{DS}_{2}$-VASc-Score 3,4 sowie HAS-BLED-Score 2,0) war die Altersund Geschlechtsverteilung der Patienten in $\mathrm{D}-\mathrm{A}-\mathrm{CH}$ ähnlich, die beiden Scores lagen jedoch in D-A-CH etwas höher [17].

Der Schwerpunkt unserer Studie lag auf der Erfassung der medikamentösen Maßnahmen zur Schlaganfallprävention. Orale Antikoagulanzien niedergelassenen Bereich mit Einschluss im Jahr
Tab. 3 Antikoagulation und antithrombotische Therapie NOAK: neue nicht-VKA orale Antikoagulanzien. TAH:Thrombozytenaggregationshemmer VKA: Vitamin-K-Antagonisten. 
Abb. 1 Antikoagulanzien und Thrombozytenaggregationshemmer bei Einschluss, nach Risikoprofil ( $\mathrm{CHA}_{2} \mathrm{DS}_{2}$-VASc-Score) Die Abbildung basiert auf Patienten, bei denen die Angaben für die Berechnung des Scores ausreichten. Für 118 Patienten konnte kein $\mathrm{CHA}_{2} \mathrm{DS}_{2}$-VASc-Score berechnet werden.

NOAK: Nicht-Vitamin-K orale Antikoagulanzien;

TAH: Thrombozytenaggregationshemmer; VKA: Vitamin-K-Antagonisten.

Abb. 2 Antikoagulanzien und Thrombozytenaggregationshemmer bei Einschluss, nach Risikoprofil (HAS-BLED-Score)

Die Abbildung basiert auf Patienten, von denen die Angaben für die Berechnung des Scores ausreichten. NOAK: Nicht-Vitamin-K orale Antikoagulanzien;

TAH: Thrombozytenaggregationshemmer; VKA: Vitamin-K-Antagonisten. sind hierbei sehr effektiv: Hart et al. [11] berichteten auf der Grundlage einer Meta-Analyse von 29 Studien mit 28044 Patienten mit vorangegangenem Schlaganfall, dass dosisadaptiertes Warfarin das Schlaganfallrisiko um etwa $60 \%$ vermindert. In früheren Studien, z. B. im Euro Heart Survey 2003-2004 [22] und dem Register des AFNET [21] wurde eine orale Antikoagulation (OAK) bei etwa $70 \%$ der Patienten mit entsprechender Indikation eingesetzt. In der Folgezeit wurden in Deutschland etwa im ATRIUM-Register (83,3\% adäquate Antikoagulation) im hausärztlichen Bereich etwas höhere Raten berichtet $[2,19]$ - im Gegensatz zu anderen europäischen Ländern (RECORD-AF [16], GARFIELD [13, 14]). In PREFERin-AF lag die Rate der mit VKA oder NOAK behandelten Patienten bei 79,7\% bzw. 11,6\% und somit etwa in der Größenordnung von ATRIUM.

Die Qualität der INR-Einstellung wurde in PREFERin-AF durch die Bewertung der letzten 3 INRWerte vor dem Einschluss beurteilt, wobei eine adäquate INR-Kontrolle bei 2 oder 3 von 3 der letz- ten verfügbaren Werten innerhalb des Zielbereichs von 2,0-3,0 dokumentiert wurde. Diese Bedingung erfüllten $75,1 \%$ der Patienten, bei denen die entsprechenden Werte vorlagen. Insgesamt entspricht der Anteil von adäquat eingestellten VKA-Patienten in unserer Studie den vorliegenden Erfahrungswerten [17]: Wallentin et al. [31] berichteten in einer Analyse der RE-LY-Studie, dass die adäquate INR-Kontrolle (time in therapeutic range, TTR) in den 44 ausgewerteten Ländern zwischen $44 \%$ und $77 \%$ lag (Werte für D-A-CH 66$68 \%$ ) [31]. Die Methoden zur Bestimmung der TTR war jedoch eine andere (Rosendaal-Methode [25]), ebenso wie das Studiendesign (randomisierte Studie vs. Beobachtungsstudie), so dass diese Werte nicht direkt verglichen werden können. Darüber hinaus lagen in unserer Subgruppenanalyse der PREFER-in-AF bei einer Vielzahl der Patienten keine Werte vor, so dass die Ergebnisse insgesamt mit Vorsicht zu interpretieren sind.

Eine genaue Risiko-Stratifizierung der Patienten bzgl. der zu verwendenden Antikoagulation wur-
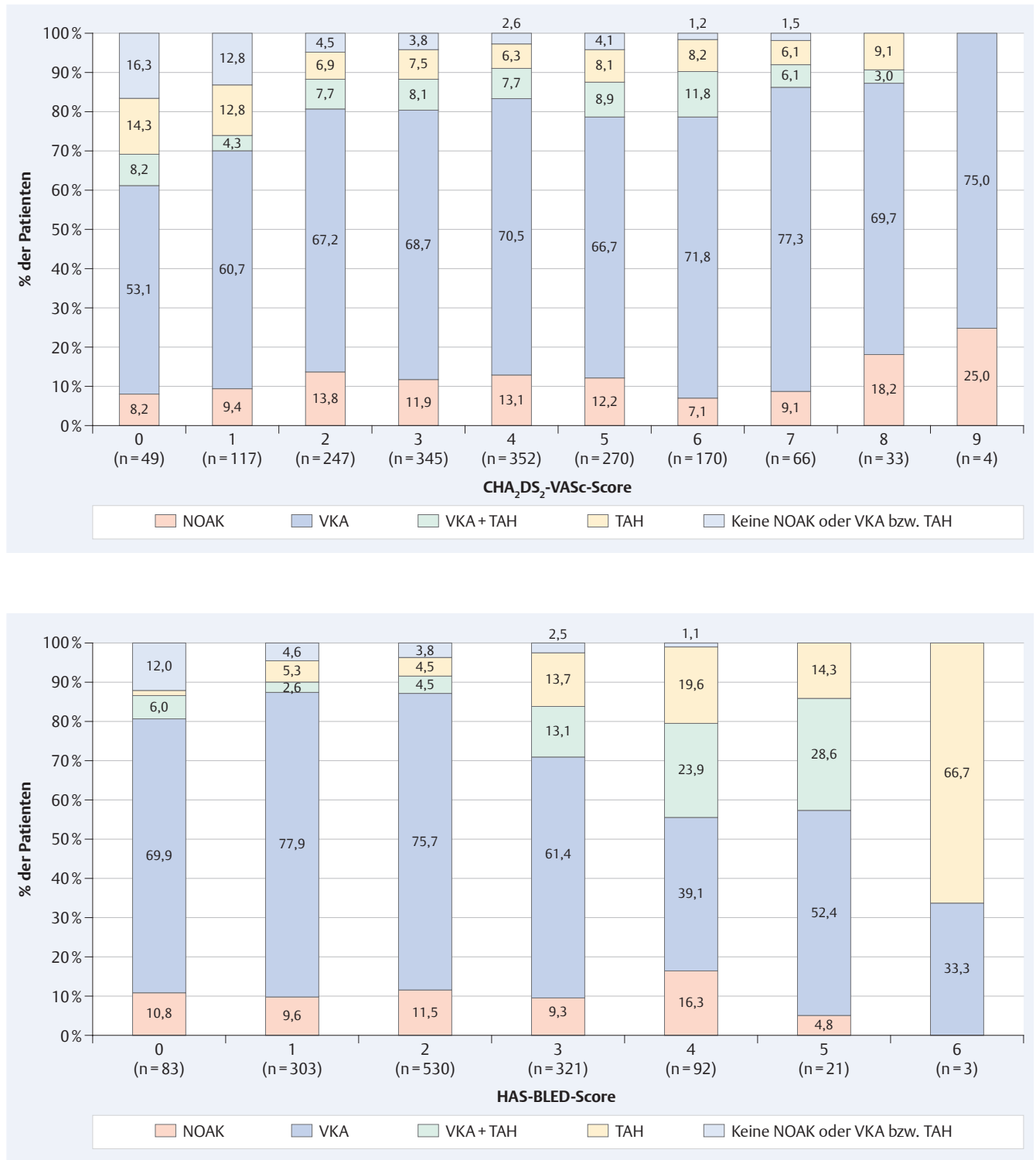
de auf den ersten Blick nur unzureichend vorgenommen: Über $60 \%$ der Patienten mit einem $\mathrm{CHA}_{2} \mathrm{DS}_{2}$-VASc-Score von 0 bzw. von 1 (und somit keiner oder nur einer relativen Indikation für eine OAK), erhielten VKA oder NOAK. Auf der anderen Seite war der Anteil der Patienten beträchtlich $(9,4 \%)$, die ein substantielles Risiko $\left(\mathrm{CHA}_{2} \mathrm{DS}_{2}\right.$ VASc-Score $\geq 4$ ) aber keine adäquate Antikoagulation hatten. Insgesamt nahm aber der Anteil der Patienten ohne OAK-Behandlung mit höheren Score-Werten ab.

Die Gründe für diese Diskrepanzen sind nicht abschließend geklärt. So ist nicht bekannt, inwieweit die Ärzte Risikoscores für ihre Patienten in der Praxis (außerhalb von klinischen Studien) tatsächlich anwenden, oder ob sie das Risiko subjektiv und orientierend abschätzen. Für die übermäßige Verschreibung einer OAK-Therapie bei niedrigen $\mathrm{CHA}_{2} \mathrm{DS}_{2}$-VASc-Scores könnten passagere Gründe für eine Antikoagulation vorliegen, etwa eine geplante oder kürzlich durchgeführte Kardioversion oder Katheterablation, die in PREFERin-AF nicht in allen Fällen erfasst wurden.

Zwischen den Ergebnissen verschiedener Scores und der ärztlichen Beurteilung können die Unterschiede gravierend sein, wie vor kurzem für Patienten mit koronarer Herzkrankheit gezeigt wurde [29]. So ist beispielsweise anzunehmen, dass behandelnde Ärzte bei diesen Niedrigrisikopatienten die Gefahr einer Blutung deutlich geringer einschätzten, als das Risiko eines Schlaganfalls. Zweitens ist es denkbar, dass Ärzte gerade bei den Patienten im hohen Alter auf eine Antikoagulation verzichten, um schwere und insbesondere intrakranielle Blutungen zu vermeiden [30]. Dies ist insbesondere der Fall, wenn das Sturzrisiko der Patienten als wesentlich angesehen wird $[8,27]$. Hylek et al. [12] berichteten, dass Patienten im Alter von 70-79 Jahren in 59\% und Patienten ab 90 Jahren nur in $24 \%$ der Fälle eine Antikoagulation verordnet wurde. Auch im AFNET wurde nur $45 \%$ der Patienten zwischen 80 und 90 Jahren und 15\% der Patienten über 90 Jahre eine Antikoagulation verschrieben [21]. Dies steht im Gegensatz zu den Daten aus klinischen Studien, wonach der „Nettonutzen“ einer Therapie mit OAK gerade bei Patienten im höheren Alter die Risiken überwiegt [11, 18]. Entsprechend wird auch in den aktuellen Leitlinien klar formuliert, dass ein hohes Blutungsrisiko (wie beispielsweise im HAS-BLEDScore berechnet) nicht dazu führen sollte, Patienten mit Indikation für eine blutgerinnungshemmende Therapie diese a priori zu verweigern.

Obwohl die Kombination von Plättchenhemmern und Antikoagulanzien zu einer erhöhten Blutungsneigung führt, wird eine solche Kombinationstherapie auch bei Patienten mit mutmaßlich stabiler vaskulärer Erkrankung häufig angewandt. Im AFNET-Register erhielten in Abhängigkeit vom VHF-Typ 16-26\% und in ATRIUM 28\% der Patienten TAH. In PREFER-in-AF wurden $22 \%$ der Patienten ausschließlich mit TAH behandelt und weitere $10 \%$ erhielten sie in Kombination zu anderen OAK. Die (alleinige) Behandlung mit Acetylsalicylsäure und anderen TAH reduziert das Schlaganfallrisiko nur um etwa 20\% [11]. Auch die BAFTA-Studie [18] und die aktuelle AVERROES-Studie belegen, dass TAH einer oralen Antikoagulation unterlegen sind (bei häufig unterschätztem Risiko für schwere Blutungen) und daher für den ganz überwiegenden Teil der Patienten keine Therapiealternative zur OAK darstellen [7]. Bei Anwendung der TAH in der Kombinationstherapie zusätzlich zu OAK ist insbesondere beim Vorliegen von Risikofaktoren wie weiblichem Geschlecht, Diabetes mellitus, höherem Alter, Alkoholmissbrauch oder Anämie das Risiko für Blutungen erhöht [28].

Limitationen | Bei der Planung und Initiierung des Registers wurde darauf geachtet, dass die teilnehmenden Zentren für die Situation in den teilnehmenden Ländern repräsentativ waren. Bei den Patienten sollte durch den Verzicht auf Ausschluss-

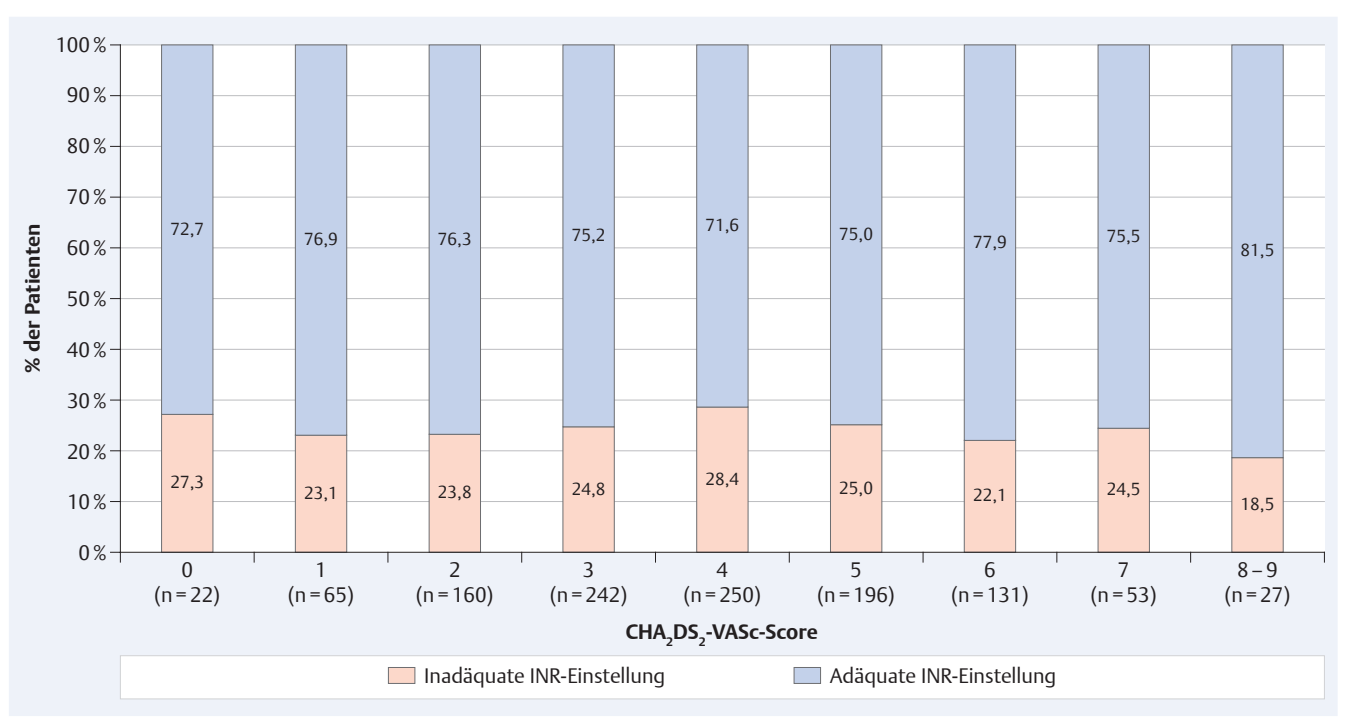

Abb. 3 Qualität der INR-Einstellung nach VHF-Typ und Risikoprofil ( $\mathrm{CHA}_{2} \mathrm{DS}_{2}$-VASc-Score) Die INR-Einstellung bei Patienten unter VKA wurde als adäquat definiert, wenn von den letzten 3 verfügbaren INR-Werten (in den letzten 12 Monaten vor Dokumentationsbeginn) mindestens 2 im Zielbereich 2,0-3,0 lagen. Patienten mit fehlenden Daten zur INR bzw. ohne ausreichende Angaben zur Berechnung des Scores wurden von der Analyse ausgeschlossen. 
kriterien eine Selektion (wie in klinischen Studien üblich) vermieden werden. Dennoch kann ein Bias durch Selektion nicht ausgeschlossen werden: Es ist naheliegend, dass wenig erfahrene oder mit den Leitlinien unzureichend vertraute Praxen nicht an Studien teilnehmen. Auch nehmen bestimmte Patienten nicht an Studien teil, beispielsweise bei kognitiver Einschränkung, Sprachproblemen oder bei schlechtem Allgemeinzustand [6]. Allerdings zeigte unsere Studie hinsichtlich der Patientencharakteristika und der Anwendung von VKA ähnliche Ergebnisse wie andere etwa im selben Zeitraum durchgeführte Studien aus Deutschland im niedergelassenen Bereich (MOVE bei Kardiologen [2] und ATRIUM bei Allgemeinärzten [19]). In PREFER-in-AF nahmen vorrangig Kardiologen teil, die im Vergleich zu anderen Fachrichtungen öfter Antikoagulanzien verordnen [6].

\section{Konsequenz für Klinik und Praxis}

- Die Zahl der Patienten mit Vorhofflimmern, die mit oralen Antikoagulanzien (VKA oder NOAK) behandelt wurden, war in der PREFER-in-AF-Studie sehr hoch.

- Allerdings entsprach die Therapie nicht immer dem in den Leitlinien empfohlenem Behandlungsprinzip. Es muss also davon ausgegangen werden, dass die RisikoStratifizierung der Patienten in Bezug auf die Antikoagulation nur unzureichend vorgenommen wurde.

- $12 \%$ der Patienten wurden im Beobachtungszeitraum (Januar 2012 - Januar 2013) mit einem NOAK behandelt.

\section{Danksagung}

Josef Schmitt von Daiichi Sankyo Europe beriet die Studiengruppe bezüglich der statistischen Auswertung und der Programmierung. Die Autoren danken den teilnehmenden Studienärzten und den Praxis- / Klinikmitarbeitern für ihre Unterstützung der Studie. Die Autoren danken darüber hinaus David Pittrow (3P Consulting, Seefeld) für die Unterstützung bei der Verfassung des Manuskripts.

\section{Interessenkonflikte}

JS: Beratungs- und/ oder Vortragshonorare: Amgen, AstraZeneca, Atricure, Bayer, Biosense Webster, Biotronik, Boehringer-Ingelheim, Boston Scientific, Bristol-Myers Squibb, Cook Medical, Daiichi Sankyo, Medtronic, Novartis, Pfizer, Roche, Sanofi-Aventis, Sorin und St. Jude Medical; Grant Support für seine Institution: Bayer Healthcare, Biotronik, Daiichi Sankyo, Medtronic und St. Jude Medical. JS ist Co-director von CorXL.

MG: Vortrags- und/ oder Beratertätitgkeit: Bayer, Boehringer-Ingelheim, Novartis, Boston Scientific, Bristol-Myers Squibb, Daiichi-Sankyo, Boston Scientific, Biotronik, Medtronic, St. Jude Medical. HD: Mitglied des Steering Committee und deutscher Koordinator für die Studien Germany RE-LY, APPRAISE1 und -2, GARFIELD-Register und PREFER-in-AF. Honorare und Forschungsunterstützung: AstraZeneca, Bayer, Berlin-Chemie, Boehringer-Ingelheim, Boston Scientific, Bristol-Myers Squibb, DaiichiSankyo, Lilly, MSD Sharp\&Dohme, BMFT, Harvard Med. es. Inst., Thrombosis Research Institute.
BA: frühere Mitarbeiterin von Daiichi-Sankyo, München.

MWHM: Mitarbeiter von Daiichi-Sankyo, München. PK: Beraterhonorare: AstraZeneca, Bayer, BoehringerIngelheim, Bristol-Myer Squibb, Daiichi-Sankyo, $3 \mathrm{M}$ Medica, MEDA Pharma, Medtronic, Merck, Otsuka, Pfizer, Sanofi-Aventis, Servier, Siemens, Takeda; Forschungsunterstützung: Cardiovascular Therapeutics, European Union, Fondation LeDucq, Bundesministerium für Bildung und Forschung, Deutsche Forschungsgemeinschaft, 3 M Medica, MEDA Pharma, Medtronic, OMRON, St. Jude Medical.

\section{Finanzierung}

Die PREFER-in-AF-Studie wurde von Daiichi Sankyo Europe $\mathrm{GmbH}$ finanziert. Die Mitglieder des Steering Board erhielten Honorare für Beratungsleistungen im Rahmen der Studie.

\section{Literatur}

1 Albertsen IE, Rasmussen LH, Overvad TF et al. Risk of stroke or systemic embolism in atrial fibrillation patients treated with warfarin: a systematic review and meta-analysis. Stroke 2013; 44: 1329-1336

2 Bonnemeier H, Bosch RF, Kohlhaussen A et al. Presentation of atrial fibrillation and its management by cardiologists in the ambulatory and hospital setting: MOVE cross-sectional study. Curr Med Res Opin 2011; 27: 995-1003

3 Camm A, Lip G, Atar D et al. 2012 Focused Update of the ESC Guidelines on the Management of Atrial Fibrillation. Eur Heart | 2012; 33: 2719-2747

4 Camm AJ, Kirchhof P, Lip GY et al. Guidelines for the management of atrial fibrillation: The Task Force for the Management of Atrial Fibrillation of the European Society of Cardiology (ESC). Eur Heart J 2010; 31: 2369-2429

5 Connolly S], Ezekowitz MD, Yusuf S et al. Dabigatran versus warfarin in patients with atrial fibrillation. N Engl ] Med 2009; 361: 1139-1151

6 Delgado-Rodriguez M, Llorca J. Bias. J Epidemiol Community Health 2004; 58: 635-641

7 Diener HC, Eikelboom J, Connolly SJ et al. Apixaban versus aspirin in patients with atrial fibrillation and previous stroke or transient ischaemic attack: a predefined subgroup analysis from AVERROES, a randomised trial. Lancet Neurol 2012; 11: 225-231

8 Donze J, Clair C, Hug B et al. Risk of falls and major bleeds in patients on oral anticoagulation therapy. Am J Med 2012; 125: 773-778

9 Granger CB, Alexander JH, McMurray IJ et al. Apixaban versus warfarin in patients with atrial fibrillation. N Engl J Med 2011; 365: 981-992

10 Hart RG, Pearce LA. Current status of stroke risk stratification in patients with atrial fibrillation. Stroke 2009; 40: 2607-2610

11 Hart RG, Pearce LA, Aguilar MI. Meta-analysis: antithrombotic therapy to prevent stroke in patients who have nonvalvular atrial fibrillation. Ann Intern Med 2007; 146: 857-867

12 Hylek EM, D'Antonio J, Evans-Molina C et al. Translating the results of randomized trials into clinical practice: the challenge of warfarin candidacy among hospitalized elderly patients with atrial fibrillation. Stroke 2006; 37: 1075-1080

13 Kakkar AK, Mueller I, Bassand JP et al. Risk profiles and antithrombotic treatment of patients newly diagnosed with atrial fibrillation at risk of stroke: perspectives from the international, observational, prospective GARFIELD registry. PloS one 2013; 8: e63479

14 Kakkar AK, Mueller I, Bassand JP et al. International longitudinal registry of patients with atrial 
fibrillation at risk of stroke: Global Anticoagulant Registry in the FIELD (GARFIELD). Am Heart ] 2012; 163: 13-19

15 Kirchhof P, Ammentorp B, Darius H et al. Management of atrial fibrillation in seven European countries after the publication of the 2010 ESC Guidelines on atrial fibrillation: primary results of the PREvention of thromboemolic events--European Registry in Atrial Fibrillation (PREFER-in-AF). Europace 2014; 16: 6-14

16 Le Heuzey J, Breithardt G, Camm J et al. The RecordAF Study: Design, Baseline Data, and Profile of Patients According to Chosen Treatment Strategy for Atrial Fibrillation. Am J Cardiol 2010; 105: 687-693

17 Le Heuzey JY, Ammentorp B, Darius H et al. Differences among western European countries in anticoagulation management of atrial fibrillation. Data from the PREFER-IN-AF registry. Thromb Haemost 2014; 111: 833-841

18 Mant J, Hobbs FD, Fletcher $\mathrm{K}$ et al. Warfarin versus aspirin for stroke prevention in an elderly community population with atrial fibrillation (the Birmingham Atrial Fibrillation Treatment of the Aged Study, BAFTA): a randomised controlled trial. Lancet 2007; 370: 493-503

19 Meinertz T, Kirch W, Rosin L et al. Management of atrial fibrillation by primary care physicians in Germany: baseline results of the ATRIUM registry. Clin Res Cardiol 2011; 100: 897-905

20 Mozaffarian D, Benjamin EJ, Go AS et al. Heart Disease and Stroke Statistics-2015 Update: A Report From the American Heart Association. Circulation 2015; 131: e29-e322

21 Nabauer M, Gerth A, Limbourg T et al. The Registry of the German Competence NETwork on Atrial Fibrillation: patient characteristics and initial management. Europace 2009; 11: 423-434
22 Nieuwlaat R, Capucci A, Camm AJ et al. Atrial fibrillation management: a prospective survey in ESC Member Countries: The Euro Heart Survey on Atrial Fibrillation. Eur Heart ] 2005; 26: 2422-2434

23 Ntaios G, Papavasileiou V, Diener HC et al. Nonvitamin-K-antagonist oral anticoagulants in patients with atrial fibrillation and previous stroke or transient ischemic attack: a systematic review and meta-analysis of randomized controlled trials. Stroke 2012; 43: 3298-3304

24 Patel MR, Mahaffey KW, Garg J et al. Rivaroxaban versus Warfarin in Nonvalvular Atrial Fibrillation. N Engl J Med 2011; 365: 883-891

25 Rosendaal FR, Cannegieter SC, van der Meer F], Briet $\mathrm{E}$. A method to determine the optimal intensity of oral anticoagulant therapy. Thromb Haemost 1993; 69: 236-239

26 Savelieva I, Camm A]. 'Preferred' management of atrial fibrillation in Europe. Europace 2014; 16: 1-3

27 Sellers MB, Newby LK. Atrial fibrillation, anticoagulation, fall risk, and outcomes in elderly patients. Am Heart J 2011; 161: 241-246

28 Shireman TI, Mahnken JD, Howard PA et al. Development of a contemporary bleeding risk model for elderly warfarin recipients. Chest 2006; 130: 1390-1396

29 Silber S, Jarre F, Pittrow D et al. Kardiovaskuläre Risikoabschätzung in der Hausarztpraxis (DETECT). Med Klin (Munich) 2008; 103: 638-645

30 Torn M, Bollen WL, van der Meer FJ et al. Risks of oral anticoagulant therapy with increasing age. Arch Intern Med 2005; 165: 1527-1532

31 Wallentin L, Yusuf S, Ezekowitz MD et al. Efficacy and safety of dabigatran compared with warfarin at different levels of international normalised ratio control for stroke prevention in atrial fibrillation: an analysis of the RE-LY trial. Lancet 2010; 376: 975-983
DOI 10.1055/s-0041-108996

Dtsch Med Wochenschr 2015; 140: e247-e255 (c) Georg Thieme Verlag KG . Stuttgart · New York . ISSN 0012-0472 